\title{
Distributional Limits of Renormalized Feynman Integrals with Zero-Mass Denominators
}

\author{
J. H. Lowenstein * \\ Department of Physics, New York University, New York, N.Y. 10003, USA \\ E. Speer $\star \star$ \\ Department of Mathematics, Rutgers University, New Brunswick, N.J. 08903, USA
}

\begin{abstract}
It is shown that the $\varepsilon \rightarrow 0$ limits of renormalized Feynman integrals exist and define Lorentz invariant tempered distributions in the external momenta. The proof applies to the case where some or all particle masses vanish.
\end{abstract}

Within the Bogoliubov-Parsiuk-Hepp-Zimmermann (BPHZ) framework of renormalized perturbation theory [1-3], the connected Green functions of elementary and composite fields are expressed as sums of contributions from Feynman diagrams, each of which corresponds to a subtracted momentum-space integral of the form

$$
J_{\varepsilon}(p)=\int_{\mathbb{R}^{4 M}} d k R_{\varepsilon}(p, k)
$$

$p=\left(p_{1}, p_{2}, \ldots, p_{N}\right)=$ independent external momenta $\left(p_{i} \in \mathbb{R}^{4}\right), k=\left(k_{1}, k_{2}, \ldots, k_{N}\right)=$ independent internal (loop) momenta $\left(k_{j} \in \mathbb{R}^{4}\right)$.

With Zimmermann's subtraction prescription [3], if all mass parameters are positive, the integral (1) converges absolutely for all $\varepsilon>0$; moreover, as $\varepsilon$ tends to zero, $J_{\varepsilon}(p)$ approaches, in the sense of tempered distributions, a Lorentz invariant limit [4]. Zimmermann's proof of the distributional limit was based on an earlier theorem of Hepp [2]. There, also, the non-vanishing of all masses was a crucial hypothesis.

In Ref. [5], one of us (J.H.L.) introduces a modified subtraction scheme such that the integral

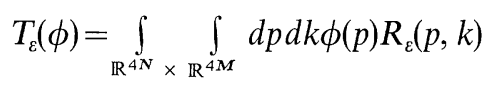

converges absolutely for arbitrary $\varepsilon>0$ and $\phi \in \mathscr{S}\left(\mathbb{R}^{4 N}\right)$, provided that a certain infrared power-counting criterion is fulfilled. There is no requirement that any of the masses of the unsubtracted integrand be positive (there is, however, at least one non-zero normalization mass appearing in subtractions terms). In the present article, we use the absolute convergence of (2) to show that $T_{\varepsilon}$ approaches, when $\varepsilon$ tends to zero, a Lorentz invariant limit as a tempered distribution. Again, some

* Research supported in part by the National Science Foundation Grant MPS-74-21778.

$\star \star$ Research supported in part by the National Science Foundation Grant GP-43758. 
of the masses may vanish. Our proof shares some of the features of Hepp's proof of the purely massive case in $[2,6]$, and our technique of integration by parts is similar to that of [7].

Our main theorem (Theorem 1 below), combined with the subtraction prescriptions of $[5,8]$ and the absolute convergence theorems of $[5,9,10]$, allow one to construct, within the BPHZ framework, the perturbative Green functions of a wide class of models involving zero-mass particles. This can now be done with the same level of mathematical precision attained by Blanchard and Seneor [11] using the method of Epstein and Glaser [12]. Normal product techniques based on $[5,8]$ have already proven extremely useful in deriving Ward identities and other structural relations in a number of theories with zero-mass particles $[8,12-15]$.

We begin with a (presumably subtracted) momentum-space Feynman integrand

$$
R_{\varepsilon}(p, k)=P(p, k, \varepsilon) / \prod_{i=1}^{n}\left(\bar{l}_{i}^{2}-\bar{\mu}_{i}^{2}\right) .
$$

$P$ is a polynomial,

$$
l_{i}=\sum_{j=1}^{N} \gamma_{i j} p_{j}+\sum_{j=1}^{M} \beta_{i j} k_{j}
$$

a linear form in the momenta, and the $\varepsilon$ dependence in the denominators is included via Zimmermann's prescription [3]:

$$
\begin{aligned}
\bar{l}_{i}^{2} & =\left(l_{i}^{0}\right)^{2}-l_{i}^{2}(1-i \varepsilon), \\
\mu_{i}^{2} & =\mu_{i}^{2}(1-i \varepsilon),
\end{aligned}
$$

with $\mu_{i}^{2} \geqq 0$. We assume that $\operatorname{rank} \beta=M$.

Our main result is

Theorem 1. Suppose that, for $\varepsilon>0, R_{\varepsilon}$ defines an element in $\mathscr{S}^{\prime}\left(\mathbb{R}^{4 N}\right)$ via an integral (2) which is absolutely convergent for every $\phi \in \mathscr{S}\left(\mathbb{R}^{4 N}\right)$. Then

$$
T_{0}=\lim _{\varepsilon \rightarrow 0+} T_{\varepsilon}
$$

exists in $\mathscr{S}^{\prime}\left(\mathbb{R}^{4 N}\right) ; T_{0}$ is a distribution of order at most $n-2 M-[r / 2]$, where $r$ is the degree of $P$ in $k$. If $P(p, k, 0)$ is a Lorentz covariant polynomial, then $T_{0}$ is a distribution of the same Lorentz covariance.

The first step in proving Theorem 1 is to introduce Feynman parameters, so that (2) becomes

$$
T_{\varepsilon}(\phi)=(n-1) ! \int d p d k \phi(p) P(p, k, \varepsilon) \int_{\mathscr{D}} d \alpha\left[\sum_{i=1}^{n} \alpha_{i}\left(\bar{l}_{i}^{2}-\bar{\mu}_{i}^{2}\right)\right]^{-n},
$$

where $\mathscr{D}=\left\{\alpha \mid \alpha_{i} \geqq 0, \sum \alpha_{i}=1\right\}$. Zimmermann [3] has shown that (4) is absolutely convergent; his proof applies to the case considered here in which some $\mu_{i}$ may vanish. By Fubini's theorem, we may then interchange the $\alpha$ and $k$ integrations. The $k$ integrations are evaluated (for almost every $\alpha$ ) by completing the square 
and diagonalizing the resulting quadratic form in the denominator (it is here we need $\operatorname{rank} \beta=M)$. We find

$$
T_{\varepsilon}(\phi)=(1-i \varepsilon)^{-\left(3 M / 2+\left[r^{\prime} / 2\right]\right)} I_{\varepsilon}^{1}(\phi),
$$

where $r^{\prime}$ is the degree of $P$ in $\boldsymbol{k}$, and

$$
I_{\varepsilon}^{1}=\int_{\mathbb{R}^{4 N}} d p \int_{\mathscr{D}} d \alpha N(\alpha, p, \varepsilon) \phi(p) F_{\varepsilon}^{1}(\alpha, p)^{-t} .
$$

Here $N(\alpha, p, \varepsilon)$ is a polynomial in $p$ and $\varepsilon$, rational in $\alpha$;

$$
\begin{aligned}
t & =n-2 M-[r / 2] ; \\
F_{\varepsilon}^{1}(\alpha, p) & =\sum_{i, j=1}^{N} p_{i}^{0} A_{i j} p_{j}^{0}-\left(\sum_{i, j=1}^{n} \sum_{\mu=1}^{3} p_{i}^{\mu} A_{i j} p_{j}^{\mu}+M^{2}\right)(1-i \varepsilon) \\
& =p^{0} A p^{0}-\left(\boldsymbol{p} A \boldsymbol{p}+M^{2}\right)(1-i \varepsilon) ; \\
M^{2} & =\sum_{i=1}^{n} \alpha_{i} \mu_{i}^{2} ;
\end{aligned}
$$

and $A$ is an $N \times N$ quadratic form, rational in $\alpha$, continuous in $\mathscr{D}$, and positive definite when all $\alpha_{i}$ are positive. We note that, if $P(p, k, 0)$ is Lorentz covariant, so is $N(\alpha, p, 0)$. Thus Theorem 1 will follow from

Theorem 2. If the integral (5) is absolutely convergent for all $\varepsilon>0$ and all $\phi \in \mathscr{S}\left(\mathbb{R}^{4 N}\right)$, then

$$
I_{0}=\lim _{\varepsilon \rightarrow 0+} I_{\varepsilon}^{1}
$$

exists in $\mathscr{S}^{\prime}\left(\mathbb{R}^{4 N}\right) ; I_{0}$ is a distribution of order at most $t$. If $N(p, k, 0)$ is Lorentz covariant, so is $I_{0}$.

Theorem 2 can be expected to have independent application to $\alpha$-space renormalization methods.

Our proof of the Lorentz covariance is similar to that of Zimmermann [3]: we show that $I_{0}$ is also the limit of distributions which are manifestly covariant. Thus define

$$
\begin{aligned}
F_{\varepsilon}^{2} & =p A p-M^{2}+i \varepsilon \\
I_{\varepsilon}^{2}(\phi) & =\int d p \int_{\mathscr{D}} d \alpha N(\alpha, p, \varepsilon) \phi(p) F_{\varepsilon}^{2}(\alpha, p)^{-t} ;
\end{aligned}
$$

$F_{\varepsilon}^{2}$ is Lorentz invariant. We will write $F_{\varepsilon}^{i}=p A p+i \varepsilon Q^{i}(i=1,2)$, where

$$
Q^{i}=\left\{\begin{array}{ll}
\boldsymbol{p} A \boldsymbol{p}+M^{2} & i=1 \\
1 & i=2
\end{array} .\right.
$$

Lemma 1. The integral (6) is absolutely convergent for all $\varepsilon>0$ and all $\phi \in \mathscr{S}\left(\mathbb{R}^{4 N}\right)$.

Proof. Since $A$ is continuous on the compact set $\mathscr{D}$,

$$
\boldsymbol{p} A \boldsymbol{p}+M^{2} \leqq K\left(1+\sum\left|\boldsymbol{p}_{i}\right|^{2}\right)
$$


for some constant $K$; we may take $K \geqq 1$. Then

$$
\begin{aligned}
\left|F_{\varepsilon}^{1}(\alpha, p)\right|^{2} & =\left|p A p-M^{2}\right|^{2}+\varepsilon^{2}\left|\boldsymbol{p} A \boldsymbol{p}+M^{2}\right| \\
& \leqq\left(\left|p A p-M^{2}\right|+\varepsilon^{2}\right) K\left(1+\sum\left|\boldsymbol{p}_{i}\right|^{2}\right)=\left|F_{\varepsilon}^{2}(\alpha, p)\right|^{2} K\left(1+\sum\left|\boldsymbol{p}_{i}\right|^{2}\right) .
\end{aligned}
$$

Since

$$
\psi(p)=\phi(p)\left[K\left(1+\sum\left|\boldsymbol{p}_{i}\right|^{2}\right)\right]^{t / 2}
$$

is in $\mathscr{S}\left(\mathbb{R}^{4 N}\right)$, the integrand for $I_{\varepsilon}^{2}(\phi)$ is dominated by the absolute value of the integrand for $I_{\varepsilon}^{1}(\psi)$.

Lemma 2. Let $N(\alpha, p, \varepsilon)=\sum_{r, a \geqq 0} N_{r a}(\alpha, p) \varepsilon^{r}$, where $N_{r a}$ is homogeneous of degree a in $p^{0}=\left(p_{1}^{0}, \ldots, p_{N}^{0}\right)$, and let

$$
J_{r a \varepsilon}^{i}=\int d p \int_{\mathscr{D}} d \alpha N_{r a}(\alpha, p) \phi(p) F_{\varepsilon}^{i}(\alpha, p)^{-t},
$$

$i=1,2$. Then (7) is absolutely convergent.

Proof. For fixed $\lambda>0$, we make the variable charge $\left(p_{j}^{0}, \boldsymbol{p}_{j}\right)=\left(\lambda q_{i}^{0}, \boldsymbol{q}_{i}\right)$ in (5), to obtain

$$
I_{\varepsilon}^{i}=\int d \alpha d q \sum_{a, r} \lambda^{N+a} \varepsilon^{r} N_{r a}(\alpha, q) \tilde{\phi}(q) F_{\varepsilon}^{i}(\alpha, p(q))^{-t},
$$

where $\tilde{\phi}(q)=\phi(p)$ defines $\tilde{\phi} \in \mathscr{S}^{\prime}\left(\mathbb{R}^{4 N}\right)$. Note that for $\lambda, \varepsilon$, and $\delta$ in a fixed compact subset of $R^{+}=\{x \mid x>0\}$, there is a constant $K$ with

$$
|(\lambda x-1+i \varepsilon) /(x-1+i \delta)|<K
$$

for all $x \in \mathbb{R}$. Thus

$$
\left|F_{\varepsilon}^{1}(\alpha, p(q)) / F_{\delta}^{1}(\alpha, q)\right|=|(\lambda D-1+i \varepsilon) /(D-1+i \delta)|<K
$$

for almost every $\alpha, p$, where

$$
D(\alpha, p)=p^{0} A p^{0} /\left(\boldsymbol{p} A \boldsymbol{p}+M^{2}\right) .
$$

Since (8) is absolutely convergent, (9) implies that

$$
\int d \alpha d q \sum_{a, r} \lambda^{N+a} \varepsilon^{r} N_{r a}(\alpha, q) \tilde{\phi}(q) F_{\delta}^{1}(\alpha, q)^{-t}
$$

is absolutely convergent for fixed $\delta>0$ and $\lambda, \varepsilon$ in an open set; the integral $J_{\text {ra } \delta}^{1}$ defining the coefficient of $\lambda^{N+a} \varepsilon^{r}$ in (11) is therefore also absolutely convergent. The convergence of $J_{\text {ras }}^{2}$ now follows from Lemma 1.

Lemma 3. If the integral (7) defining $J_{r a \varepsilon}^{i}$ is absolutely convergent for all $\varepsilon>0$, then

$$
J_{r a 0}^{i}(\phi)=\lim _{\varepsilon \rightarrow 0+} J_{r a \varepsilon}^{i}(\phi)
$$

exists and defines a tempered distribution of order at most $t$; moreover, $J_{r a 0}^{1}=J_{r a 0}^{2}$. 
We will prove this lemma shortly, but first note that it implies our main result. Proof of Theorem 2.

$$
\begin{aligned}
I_{0} & \equiv \lim _{\varepsilon \rightarrow 0+} I_{\varepsilon}^{1} \\
& =\lim _{\varepsilon \rightarrow 0+} \int d \alpha d p \sum_{r, a} N_{r a} \varepsilon^{r} \phi(p) F_{\varepsilon}^{1}(\alpha, p)^{-t} \\
& =\sum_{r} \lim _{\varepsilon \rightarrow 0+} \varepsilon^{r} \int d \alpha d p \sum_{a} N_{r a} \phi(p) F_{\varepsilon}^{1}(\alpha, p)^{-t} \\
& =\lim _{\varepsilon \rightarrow 0+} \int d \alpha d p N(\alpha, p, 0) \phi(p) F_{\varepsilon}^{1}(\alpha, p)^{-t} \\
& =\lim _{\varepsilon \rightarrow 0+} \int d \alpha d p N(\alpha, p, 0) \phi(p) F_{\varepsilon}^{2}(\alpha, p)^{-t} .
\end{aligned}
$$

These limits exist as distributions of order at most $t$ by Lemma 3. Finally, if $N(\alpha, p, 0)$ is Lorentz covariant, the last equality represents $I_{0}$ as a limit of distributions with the same covariance.

Proof of Lemma 3. Let $\chi(x)$ be a $C^{\infty}$ function of $x \in \mathbb{R}$ with $0 \leqq \chi(x) \leqq 1$ and

$$
\chi(x)= \begin{cases}0, & \text { if } x<1 / 3 \\ 1, & \text { if } x>2 / 3 .\end{cases}
$$

We will write $J_{\text {ra }}^{i}=K_{\varepsilon}^{i}+L_{\varepsilon}^{i}$, where

$$
\begin{aligned}
K_{\varepsilon} & =\int d \alpha d p N_{r a}(\alpha, p) \phi(p) F_{\varepsilon}(\alpha, p)^{-t}(1-\chi(D)), \\
L_{\varepsilon} & =\int d \alpha d p N_{r a}(\alpha, p) \phi(p) F_{\varepsilon}(\alpha, p)^{-t} \chi(D) ;
\end{aligned}
$$

here $D=D(\alpha, p)$ is given by $(10)$. Now for $(1-\chi(D)) \neq 0,|D-1|>1 / 3$, and

$$
|D-1+i \varepsilon| /|D-1| \leqq 1+3 \varepsilon .
$$

\section{Hence}

$$
\begin{aligned}
\left|p A p-M^{2}\right| & =\left|\boldsymbol{p} A \boldsymbol{p}+M^{2}\right||D-1| \\
& \geqq\left|\boldsymbol{p} A \boldsymbol{p}+M^{2}\right||D-1+i \varepsilon|(1+3 \varepsilon)^{-1}=\left|F_{\varepsilon}^{1}(\alpha, p)\right|(1+3 \varepsilon)^{-1} .
\end{aligned}
$$

This inequality and the convergence of the integral (7) defining $J_{\text {rac }}^{1}$ imply that the integral

$$
K_{0}=\iint d \alpha d p N_{r a}(\alpha, p) \phi(p)\left|p A p-M^{2}\right|^{-t}[1-\chi(D)]
$$

is absolutely convergent; the Lebesgue dominated convergence theorem and the inequality

$$
\left|F_{\varepsilon}^{i}(\alpha, p)\right| \geqq\left|p A p-M^{2}\right|
$$

imply that

$$
\lim _{\varepsilon \rightarrow 0} K_{\varepsilon}^{i}=K_{0}, \quad i=1,2 .
$$

To study $L_{\varepsilon}^{i}$ we write

$$
F_{\varepsilon}^{i}(\alpha, p)^{-t}=(-1)^{t-1} 2^{-t}(t-1) !^{-1}\left[\left(p^{0} A p^{0}\right)^{-1} \sum_{i=1}^{N} p_{i}^{0} \partial / \partial p_{i}^{0}\right]^{t} \log F_{\varepsilon}^{i}(\alpha, p)
$$


If we consider (13) as an iterated integral we may insert (14) and integrate by parts with respect to $p^{0}$, to find

$$
\begin{aligned}
L_{\varepsilon}^{i}=- & 2^{-t}(t-1) !^{-1} \int d \alpha d \boldsymbol{p} \int d p^{0} \log F_{\varepsilon}^{i}(\alpha, p) \\
& \cdot\left\{\left[\sum_{i=1}^{N} \partial / \partial p_{i}^{0} p_{i}^{0} /\left(p^{0} A p^{0}\right)\right]^{t} \phi(p) N_{r a}(p, \alpha) \chi(D)\right\} .
\end{aligned}
$$

The integration by parts is justified, and hence (15) converges as an iterated integral, since for almost every $\alpha$ and $p, F_{\varepsilon}^{i}(\alpha, p)$ and $\chi(D)$ are $C^{\infty}$ functions of $p^{0}$, and the fast decrease of $\phi$ enables us to discard boundary terms.

We wish to show that (15) is in fact absolutely convergent. The quantity in brackets has the form

$$
N_{r a}\left(p^{0} A p^{0}\right)^{-t} \sum_{b} \tilde{\phi}_{b}(p) \tilde{\chi}_{b}(\alpha, p),
$$

where $\tilde{\phi}_{b} \in \mathscr{S}\left(\mathbb{R}^{4 N}\right)$ and $\tilde{\chi}_{b}$ is a bounded function with

$$
\operatorname{supp} \tilde{\chi}_{b} \subset H=\{(\alpha, p) \mid D(\alpha, p) \geqq 1 / 3\}
$$

(it is here that we use the homogeneity of $N_{r a}$ in $p^{0}$ ). Since

$$
\left|\frac{F_{\varepsilon}^{1}(\alpha, p)}{p^{0} A p^{0}}\right| \leqq 1+(1+\varepsilon) / D
$$

is bounded on $H$, the absolute convergence of $(13)$ for any $\phi \in \mathscr{S}^{\prime}\left(\mathbb{R}^{4 N}\right)$ implies that

$$
\int d \alpha d p N_{r a}(\alpha, p)\left(p^{0} A p^{0}\right)^{-t} \tilde{\phi}_{b}(p) \tilde{\chi}_{b}(p)
$$

is absolutely convergent. We will show in the Appendix (Corollary 1) that this implies that

$$
\int d \alpha d p N_{r a}\left(p^{0} A p^{0}\right)^{-t} \tilde{\phi}_{b} \tilde{\chi}_{b} \log \left|p A p-M^{2}\right|
$$

and

$$
\int d \alpha d p N_{r a}\left(p^{0} A p^{0}\right)^{-t} \tilde{\varphi}_{b} \tilde{\chi}_{b} \log \left|\boldsymbol{p} A \boldsymbol{p}+M^{2}\right|
$$

are absolutely convergent. Now we use the inequality, valid for $c>0$ and $d_{0}>d>0$,

$$
|\log (c+d)| \leqq|\log c|+\left|\log d_{0}\right|+1
$$

which implies

$$
\begin{aligned}
\left|\log F_{\varepsilon}^{i}\right| & \leqq \frac{1}{2} \log \left|F_{\varepsilon}^{i}\right|^{2}+\pi \\
& \leqq|\log | p A p+M^{2}||+\left|\log \varepsilon_{0} Q^{i}\right|+\pi+\frac{1}{2},
\end{aligned}
$$

whenever $\varepsilon \leqq \varepsilon_{0}$. Thus (15) is dominated by a fixed linear combination of $(16)-(18)$; it is absolutely convergent, and the Lebesque dominated convergence theorem implies that

$$
\lim _{\varepsilon \rightarrow 0} L_{\varepsilon}^{i}=\sum_{b} \int d \alpha d p N_{r a}\left(p^{0} A p^{0}\right)^{-t} \tilde{\varphi}_{b} \tilde{\chi}_{b} \log \left|p A p-M^{2}\right|
$$

for $i=1,2$. Finally, note that $\tilde{\varphi}_{b}$ is obtained from $\varphi$ by at most $t$ differentiations, so the limiting distribution is at order at most $t$. 


\section{Appendix}

We want to prove the statement made earlier that the addition of a logarithm to a convergent integral does not destroy convergence. We use the method of resolution of singularities, and follow the notation and ideas of [16].

Lemma 4. Let $X$ be a real analytic $n$-manifold with $f, g, h_{1}, \ldots h_{k}$ real analytic functions on $X$ and $\omega$ an analytic $n$-form on $X$. Let

$$
G=\left\{x \in X \mid h_{i}(x) \geqq 0 \text { for all } i\right\}
$$

and suppose that $G$ is compact. Then if

$$
\int_{G} f^{-1} \omega
$$

is absolutely convergent, so is

$$
\int_{G} f^{-1} \log |g| \omega .
$$

Proof. Because $G$ is compact it suffices to prove integrability in the neighborhood of every point $x_{0} \in G$. Let $t_{1}, \ldots t_{n}$ be local coordinates at $x_{0}$, so that

$$
\omega=w(t) d t_{1} \wedge \ldots \wedge d t_{n}
$$

in a neighborhood at $x$, with $w$ real analytic. We apply the Resolution Theorem [16] to resolve the set $A=\left\{t \mid f(t) g(t) w(t) \prod h_{i}(t)=0\right\}$; this produces a neighborhood $U$ of $x_{0}$, a real analytic manifold $\tilde{U}$, and a proper analytic map $\varphi: \tilde{U} \rightarrow U$ such that $\varphi:(\tilde{U}-\tilde{A}) \rightarrow U-A$ is a homeomorphism, with $\tilde{A}=\varphi^{-1}(A)$. Moreover, if $\tilde{f}=f \circ \varphi$, etc., then for any $\tilde{x} \in \tilde{U}$ and $y_{1}, \ldots y_{n}$ local coordinates centered at $\tilde{x}$ in a suitable neighborhood $\tilde{V}$ of $\tilde{x}$, each of $\tilde{f}, \tilde{g}, \tilde{h}_{i}$, and $\tilde{w}$ have the form

$$
\alpha(y) \prod_{i=1}^{n} y_{i}^{k_{2}}
$$

with $\alpha \neq 0$ in $\tilde{V}$. Finally,

$$
\varphi^{*}\left(d t_{1} \wedge \ldots \wedge d t_{n}\right)=r(y) d y_{1} \wedge \ldots \wedge d y_{n}
$$

in $\tilde{V}$, with $r(y)$ real analytic; $r(y)$ cannot vanish for $y \notin \tilde{A} \cap \tilde{V}$. Since $\varphi$ extends to a map $\varphi_{c}: U_{c} \rightarrow U_{c}$ of the complexifications, $r(y)$ is complex analytic and vanishes only on $\bigcup_{i=1}^{n}\left\{y_{i}=0\right\}$ in a complex neighborhood of $\tilde{x}$; hence $r(y)$ also has the form (20).

Now

$$
\int_{U \cap G} f^{-1} \omega=\int_{\tilde{U} \cap \varphi^{-1}(\boldsymbol{G})} \tilde{f}^{-1} \varphi^{*} \omega .
$$

The set $\varphi^{-1}(G) \cap V$ is a union of certain octants $\tilde{G}_{i}$ of $\tilde{V}$, i.e., subsets in which each $y_{i}$ has a fixed sign. Thus (19) becomes locally a sum of terms

$$
\int_{\tilde{G}_{i}} \alpha(y) \prod_{i=1}^{n} y_{i}^{j_{i}} d y_{1} \wedge \ldots \wedge d y_{n},
$$


with $\alpha(y) \neq 0$; the absolute convergence of (19) and hence (21) implies $j_{i} \geqq 0, i=1, \ldots n$. Since $\log |\tilde{g}|=\log \left|\alpha^{\prime}(y)\right|+\sum_{i=1}^{n} k_{i} \log \left|y_{i}\right|$ from (20), the inclusion of a factor $\log |\tilde{g}|$ in (21) does not affect convergence.

\section{Corollary 1. If}

$$
\int_{\mathscr{D}} d \alpha \int_{\mathbb{R}^{4 N}} d p R(\alpha, p) \phi(p) \chi_{H}(\alpha, p)
$$

converges absolutely for every $\varphi \in \mathscr{S}\left(\mathbb{R}^{4 N}\right)$, where $R$ is rational and $\chi_{H}$ is the characteristic function of $H=\{(\alpha, p) \mid D(\alpha, p)>a\}$ for $D$ rational, then so does

$$
\int_{\mathscr{D}} d \alpha \int_{\mathbb{R}^{4 N}} d p R(\alpha, p) \log |S(\alpha, p)| \phi(p) \chi_{H}(\alpha, p)
$$

where $S(\alpha, p)$ is rational.

Proof. Let

$$
B(p)=1+\sum_{i, \mu}\left|p_{i}^{\mu}\right|^{2} \text {. }
$$

It is an easy exercise to see that (22) will converge absolutely if $\phi(p)$ is replaced by $B(p)^{-r}$ for some sufficiently large $r$; moreover, it suffices to prove the convergence of (23) with this same replacement.

We define $X_{1}=\left\{\alpha \in \mathbb{R}^{n} \mid \sum \alpha_{i}=1\right\}$,

$$
X_{2}=S^{4 N}=\left\{(y, \bar{y}) \in \mathbb{R}^{4 N+1} \mid \sum_{i=1}^{N} \sum_{\mu=0}^{3}\left(y_{i}^{\mu}\right)^{2}+\bar{y}^{2}=1\right\}
$$

and $X=X_{1} \times X_{2}$. There is an injection $\lambda: \mathbb{R}^{4 N} \rightarrow X_{2}$ given by

$$
\lambda(p)=(2 p / B(p), 1-2 / B(p)),
$$

and an analytic $(n+4 N)$-form $\hat{\omega}$ on $X$ with

$$
(\mathbb{1} \times \lambda)^{*} \hat{\omega}=B(p)^{-s} d \alpha_{1} \wedge \ldots d \alpha_{n-1} \wedge d p_{1}^{0} \wedge \ldots \wedge d p_{N}^{3},
$$

for some $s$. Then (22) becomes

$$
\int_{G} R(\alpha, p) B(p)^{s-r} \hat{\omega}
$$

where $G \subset X, G$ the closure of $\left\{(\alpha, p) \mid \alpha_{i} \geqq 0, i=1, \ldots n, D(\alpha, p)>1 / 3\right\}$.

We can rewrite (24) in the form (19) of Lemma 5 as follows. Let

$$
\begin{aligned}
B(p)^{s-r} R(\alpha, p) & =R_{1}(\alpha, p) / R_{2}(\alpha, p) \\
D(\alpha, p) & =D_{1}(\alpha, p) / D_{2}(\alpha, p) \\
S(\alpha, p) & =S_{1}(\alpha, p) / S_{2}(\alpha, p),
\end{aligned}
$$


where $R_{i}, D_{i}$, and $S_{i}$ are polynomials; let $k$ denote the maximal degree in $p$ of these polynomials. If we define

$$
\begin{aligned}
\omega & =R_{1}(\alpha, p) B(p)^{-k} \hat{\omega}, \\
f(\alpha, p) & =R_{2}(\alpha, p) B(p)^{-k}, \\
h_{i}(\alpha) & =\alpha_{i}, \quad 1 \leqq i \leqq n, \\
h_{n+1}(\alpha, p) & =\left[D_{1}(\alpha, p)-a D_{2}(\alpha, p)\right] B(p)^{-k},
\end{aligned}
$$

then $\omega, f$, and $h_{1}, \ldots h_{n+2}$ are analytic on $X$, (24) becomes

$$
\int_{G} f^{-1} \omega
$$

and $G=\left\{(\alpha, p) \mid h_{i}(\alpha, p) \geqq 0, i=1,2, \ldots n+1\right\}$. Finally, we note

$$
\log |S(\alpha, p)|=\log \left|S_{1}(\alpha, p) B(p)^{-k}\right|-\log \left|S_{2}(\alpha, p) B(p)^{-k}\right|
$$

and apply Lemma 5, with $g=S_{1} B^{-k}$ and $g=S_{2} B^{-k}$ in turn, to deduce the absolute convergence of (23).

\section{References}

1. Bogoliubov, N.N., Shirkov,D.V.: Introduction to the theory of quantized fields. New York: Interscience 1959

2. Hepp, K.: Commun. math. Phys. 2, 301 (1966)

3. Zimmermann, W.: Commun. math. Phys. 15, 208 (1969)

4. Zimmermann, W.: Commun. math. Phys. 11, 1 (1969)

5. Lowenstein,J.H.: Convergence theorems for renormalized Feynman integrals with zero-mass denominators (to be submitted for publication)

6. Hepp, K.: Theorie de la renormalisation. Berlin-Heidelberg-New York: Springer 1969

7. Speer,E.: Generalized Feynman amplitudes. Princeton: Princeton University Press 1969

8. Lowenstein, J. H., Zimmermann, W.: Nucl. Phys. B 86, 77 (1975)

9. Gomes, M., Lowenstein, J.H., Zimmermann, W.: Commun. math. Phys. 39, 81 (1974)

10. Lowenstein, J.H., Zimmermann, W.: The power counting theorem for Feynman integrals with massless propagators. Max-Planck-Institut (München) (preprint)

11. Blanchard, P., Seneor, R.: CERN preprint TH 1420 (1971)

12. Epstein, H., Glaser, V.: CERN preprint TH 1156 (1970); TH 1344 (1971)

13. Clark, T.: Max-Planck-Institut (München) preprint MPI-PAE/Pth 27

14. Lowenstein, J. H.: Nucl. Phys. B 96, 189 (1975)

15. Clark, T., Rouet, R.: Renormalization of Gauge theories with massless propagators. Max-PlanckInstitut (München) (preprint)

16. Atiyah, M.: Commun. Pure Appl. Math. 23, 145 (1970)

Communicated by K. Symanzik 
